\title{
Interventions for Left Main Coronary Artery Disease
}

\author{
Sujatha Vipperla ${ }^{1}$ \\ ${ }^{1}$ Department of Cardiology, Indus Hospitals, Affiliated to CSI India, \\ SCAI, Visakhaptnam, Andhra Pradesh, India
}

Indian J Cardiovasc Dis Women-WINCARS 2018;3:49-60

\section{Introduction}

Unprotected left main coronary artery (ULMCA) disease constitutes 5 to $7 \%$ of the patients undergoing coronary angiography. ${ }^{1}$ It is associated with a high mortality as it supplies a large area of the myocardium-approximately $84 \%$ of the left ventricle in right dominant system and 100\% in a left dominant system. ${ }^{2}$ In CASS (Coronary Artery Surgery Study) registry, mortality in medically treated patients is as high as $63 \%$ at 5 years. ${ }^{3}$

Coronary artery bypass surgery (CABG) has been the standard management for patients with ULMCA disease. Percutaneous coronary intervention (PCI) has emerged as an attractive alternative to CABGs with the advent of drug-eluting stents (DES), development of antiplatelet pharmacology, and adjunctive imaging techniques.

Atherosclerotic plaque in left main coronary artery (LMCA) is qualitatively different from other segments of the coronary tree, with minimal necrotic core and less thin cap fibro atheroma. ${ }^{4}$ Seventy percent of significant left main (LM) lesions involve the bifurcation. Intimal atherosclerosis is located in areas of low shear stress along the lateral wall extending distally on the lateral walls of the left anterior descending (LAD) and left circumflex (LCX) arteries. Involvement of flow divider (carina) is minimal or absent. Plaque extending into the proximal LAD, LCX, or both may be seen in $90,66.4$, and $62 \%$, respectively.

\section{Current Guidelines for ULMCA Revascularization}

Although CABG is class I (level of evidence B), with growing body of evidence for ULMCA intervention, PCI in those patients with SYNTAX (Synergy between Percutaneous Coronary Intervention with Taxus and Cardiac Surgery) score $\leq 22$ has equivalent indication class I (level of evidence B) as per current European practice guidelines. ${ }^{5}$ Patients with SYNTAX scores (22-32) have a class Ila (level of evidence B) indication whereas CABG is preferred in patients with SYNTAX score $\geq 32$. AHA/ACC/SCAI (American Heart Association/ American College of Cardiology/Society of Cardiovascular Angiography and Interventions) guidelines assigned class
Address for correspondence Sujatha Vipperla, MD, DM, FIC, Department of Cardiology, Indus Hospitals, Affiliated to CSI India, SCAI, Visakhaptnam 530002, Andhra Pradesh, India (e-mail: sujasri@hotmail.com).

Ila recommendation in patients with favorable anatomy (SYNTAX $<22$ and ostial or trunk LM disease) and in patients with clinical characteristics that predict an increased risk of adverse surgical outcomes (e.g., moderate severe chronic obstructive pulmonary disease, disability from previous stroke, or previous cardiac surgery; STS-predicted risk of operative mortality $>2 \%)^{6}$

\section{Current Evidence for ULMCA Revascularization}

\section{Registry Data}

In the large, multinational, "all-comers" IRIS-MAIN (Interventional Research Incorporation Society-Left MAIN Revascularization) registry, ${ }^{7}$ over the past two decades increasingly complex patients with worsening risk-factor profiles have been recruited with improvements in concomitant medical therapy. Over time, the proportion of PCI treatment has progressively increased to approximately $61 \%$. Risk-adjusted survival, composite outcomes, and repeat revascularization have significantly improved for PCI over time but have relatively remained stable for CABG narrowing the gap in the treatment effect between PCI and CABG from the BMS (bare metal stent) period to the early DES period and then to the late DES period. The risks of mortality and the composite of death, myocardial infarction (MI), or stroke (9.1 vs. 9.7\%; hazard ratio [HR] 0.97; 95\% confidence interval [CI]: 0.81$1.17 ; p=0.75$ ) were equivalent between PCI and CABG, but the risks of repeat revascularization and major adverse cardiac and cerebrovascular events (MACCE) were higher in the PCI group than in the CABG group.

The results of DELTA-2 (Drug-Eluting Stent for Left Main Coronary Artery) registry $^{8}-$ an international, all-comers, multicenter registry-were compared with those from the historical DELTA-1 CABG cohort using propensity score stratification. The primary endpoint was the composite of death, MI, or stroke at the median time of follow-up. The distal LMCA was involved in $84.6 \%$ of the lesions. The primary endpoint of death, MI, or cerebrovascular accident was lower in the PCI DELTA-2 group compared with the historical DELTA-1 CABG cohort $(10.3 \%$ vs. $11.6 \% ; p=0.03)$ at 17 months of 
follow up. A favorable result was observed for PCI with respect to cerebrovascular accident whereas CABG was more advantageous with respect to target vessel revascularization.

\section{Randomized Trials}

The SYNTAX trial ${ }^{9}$ included a subset of 705 patients with LM disease. There was no significant difference in MACCE (death, $\mathrm{MI}$, stroke, and repeat revascularization) at 5 years $(36.9 \%$ in $\mathrm{PCI}$ patients and 31\% in CABG patients) (HR 1.23; 95\% CI: $0.95-1.59 ; p=0.12$ ). Mortality was 12.8 and $14.6 \%$ in PCI and CABG patients, respectively (HR $0.88[0.58,1.32]$; $p=0.53$ ). Stroke was significantly increased in the CABG group and repeat revascularization in the PCI arm. MACCE was similar between arms in patients with low/intermediate SYNTAX scores but significantly increased in PCI patients with high scores ( $\geq 33)$.

In the EXCEL (Evaluation of XIENCE versus Coronary Artery Bypass Surgery for Effectiveness of Left Main Revascularization) trial, ${ }^{10}$ 1,905 patients-with ULMCA disease with $\geq 70 \%$ diameter stenosis, or $\geq 50 \%$ or $\leq 70 \%$ with either (1) noninvasive evidence of LM ischemia, (2) intravascular ultrasound-derived minimal lumen area (IVUS-MLA) $\leq 6.0 \mathrm{~mm}^{2}$, or (3) fractional flow reserve $(\mathrm{FFR}) \leq 0.80$, SYNTAX score $\leq 32$, and clinical and anatomic eligibility for both PCI and CABG as agreed to by the local heart team-were randomized to PCI with fluoropolymer-based cobalt-chromium everolimus-eluting stents or CABG. The primary endpoint, the composite of death from any cause, stroke, or MI at 3 years occurred in $15.4 \%$ of patients who underwent PCI group versus $14.7 \%$ of the patients who underwent $C A B G$ group meeting the noninferiority criterion. The secondary endpoint event of death, stroke, or MI at 30 days was lower in the PCI group (4.9\% in the PCI group vs. $7.9 \%$ in the CABG group). The secondary endpoint event of death, stroke, MI, or ischemia-driven revascularization at 3 years occurred in fewer patients in PCI group. CABG patients had fewer primary endpoint events than the $\mathrm{PCI}$ group patients between 30 days and 3 years after the procedure. Revascularization rates at 3 years were higher with PCI than with CABG (12.6 vs. 7.5\%) whereas the rates of early MI and major adverse events were lower with PCI than with CABG (8.1 vs. 23.0\%).

The EXCEL trial enrolled predominantly males (76\%). Most patients were clinically at low risk (60\% had stable ischemic heart disease and most patients had normal ejection fraction). Thirty percent were diabetic. According to the sites, the SYNTAX was low $(\leq 22)$ in $60.5 \%$ of the patients and intermediate (23-32) in $39.5 \%$ of the patients. The core laboratory assigned higher scores, low in $35.8 \%$ of the patients, intermediate in $40.0 \%$, and high $(\geq 33)$ in $24.2 \%$, the group that met the exclusion criteria. Most of the patients (80.5\%) had distal left main bifurcation or trifurcation disease, and two- or three-vessel coronary artery disease was present in $51.3 \%$ of the patients. Average 1.9 lesions were treated per patient. A mean of 2.4 stents with a mean total stent length of $49.1 \mathrm{~mm}$ were implanted per patient. Femoral access was used in $73 \%$ patients, and IVUS guidance was used in $77 \%$ of patients. Off-pump CABG was performed in $29 \%$. Internal mammary artery (IMA) was used in $98.8 \%$ with bilateral IMA in $28.8 \%$ patients.

NOBLE (Nordic-Baltic-British Left Main Revascularization Study) trial ${ }^{11}$ randomized patients with significant LM lesion visually assessed stenosis diameter $\geq 50 \%$ or FFR $\leq 0.80$ and no more than three additional noncomplex lesions (additional non-LM complex lesions were chronic total occlusions, bifurcation lesions requiring two-stent strategies, calcified or tortuous lesions). A total of 1,201 patients were randomly assigned to PCI or CABG. Kaplan-Meier 5-year estimates of MACCE (death from any cause, nonprocedural MI, repeat revascularization, or stroke) were $29 \%$ for $\mathrm{PCI}$ and $19 \%$ for CABG (HR 1.48; 95\% CI: 1.11-1.96) with CABG being significantly better than PCI. Though all-cause mortality was not different, the rates of MI, revascularization, and stroke were higher in PCI patients compared with CABG patients. Repeat revascularization was higher due to de novo lesion and target non-LMCA lesion revascularization. Notably 1-year MACCE was same in both the groups. Surprisingly there was no association with SYNTAX score and MACCE.

Twenty percent of the population was female with $15 \%$ diabetics. Eighty percent had stable angina and most had preserved ejection fraction. Eighty-eight percent had distal LM lesion. Elective double stenting was used in 35\% with culotte being preferred technique. $11 \%$ of PCI cases received a first-generation DES in the LM. Kissing balloon inflation (KBI) was done in 55\%, and any ostial circumflex post-dilatation was done in $79 \%$. Complete revascularization was achieved in $92 \%$. IVUS was done pre-PCI in $47 \%$ and post-PCI in $74 \%$. CABG was done with the on-pump technique in $84 \%$, with 93\% patients receiving left internal mammary artery. The number of grafts per patient was one in $4 \%$, two in $52 \%$, three in $39 \%$, four in $4 \%$, and five in $<1 \%$ of patients.

\section{Conflicting Results of EXCEL and NOBLE}

Both were well-conducted robust trials. Both studies included similar patients with similar SYNTAX scores, and distal LM was treated in the majority. The conflicting results could be due to:

1. Differences between studies in patient assessment, risk profiles, trial process, or procedural characteristics.

2. Differences in the stent used: EXCEL used a thin strut fluropolymer-based cobalt chromium everolimuseluting stents whereas NOBLE used first-generation sirolimus-eluting Cypher stent (11\%) or the biolimus-eluting Biomatrix Flex stent (89\%), resulting in a substantial difference in stent thrombosis ( 0.7 vs. $3 \%$ ). As a result, the rate of stent thrombosis or symptomatic graft occlusion was much higher after CABG than after PCI (5.4 vs. $0.7 \%$ ) in EXCEL but similar (4 vs. 3\%) in NOBLE.

3. Strategy of stenting: Both the trials used provisional stenting approach. In approximately 75\% of patients IVUS was used. Data were not available from EXCEL regarding two-stent strategy. Thirty-five percent needed a two-stent strategy with culotte technique being used in the majority in NOBLE.

4. Differences in primary endpoint used: EXCEL used death, $\mathrm{MI}$, and stroke. Investigators included periprocedural MI. 
The EXCEL trial did not include repeat revascularization. NOBLE trial, on the other hand, used only nonprocedural $\mathrm{MI}$ in the endpoint, and repeat revascularization was part of primary composite endpoint. In the NOBLE trial, there was not much difference in target LM artery revascularization but more than twofold increase in de novo lesion revascularization in the $\mathrm{PCI}$ group with most patients undergoing repeat $\mathrm{PCI}(96 \%)$.

5. Difference in trial duration: EXCEL reported data at 3 years whereas NOBLE reported data at 5 years. Remarkably in NOBLE, the 5-year mortality was not different in PCI and CABG groups whereas death, stroke, and repeat revascularization were higher in PCI group. In fact, data from EXCEL may look similar to NOBLE if repeat revascularization is included with evaluation done at 5 years.

6. An unexplained higher risk of stroke after PCI in NOBLE, which was more than twice after PCI than CABG.

Overall, 4,594 patients from five trials (EXCEL, NOBLE, SYNTAX, PRECOMBAT, Boudriot et al) were included in the meta-analysis by Nerlekar et al, ${ }^{12}$ with $50 \%$ of patients in the metanalysis undergoing PCI using DES. The prevalence of isolated ULMCA stenosis ranged from 10 to $29 \%$, with between 55 and $80 \%$ of patients having a distal bifurcation ULMCA lesion. The primary endpoint of death, MI, and stroke was similar between PCI and CABG (odds ratio [OR] 0.97; 95\% CI: 0.79-1.17; $p=0.73$ ). As expected, CABG was associated with a significant reduction in the risk of repeat revascularization.

\section{Preprocedural Assessment and Planning for Left Main Intervention}

The LM is the largest bifurcation and provides blood supply to $>50 \%$ of the total myocardial mass. It has many unique features, which demand different technical approaches compared with non-LM bifurcations. These include the following ${ }^{13}$ :

1. The SB (side branch) is usually the LCX that most often has a large reference diameter and is angulated, making it difficult to access with guidewires. Acute occlusion of the LCX usually results in considerable ischemia and may induce acute ischemic mitral regurgitation. The T-shaped bifurcation angle of the LM may also affect implantation technique, and a highly angulated LCX takeoff may impact prognosis after LM stenting.

i. The presence of calcification in the LM is a frequent occurrence, given that the patients' mean age with LM disease is higher.

2. The LM is the only bifurcation where the proximal MB originates directly from the aorta. Possibility of guidewires to go behind LM stent struts, guide catheter distortion of stent, and stent longitudinal compression can increase complexity.

3. The proximal reference diameter may reach $>5 \mathrm{~mm}$, which is close to the dilatation limit of many coronary stents.

4. LM trifurcations are seen in approximately $10 \%$ of LM cases and may require specific treatment strategies.

\section{Indications}

Conventionally ULMCA with $\geq 50 \%$ diameter stenosis indicates hemodynamic significance needing intervention. Angiographic assessment of LMCA has inherent limitations due to short vessel segment, lack of reference vessel, eccentricity, foreshortening on angiography, and missed ostial disease due to deep catheter engagement. FFR to assess the functional significance of LMCA lesion is emerging as a complimentary modality, especially in equivocal lesions. There is a poor correlation between angiography and FFR with interobserver concordance of only $52 \%$ in one study that also found that $23 \%$ of patients had an LMCA stenosis $\leq 50 \%$ with FFR $<0.80 .{ }^{14}$

A meta-analysis of eight trials ${ }^{15}$ detected no significant difference in primary endpoint (all cause death, nonfatal MI, and revascularization) between revascularized and deferred groups. The rate of subsequent revascularization was higher in the deferred group. Whether this was due to LMCA intervention was not reported. As underlying plaque composition rather than hemodynamic significance of a lesion that determines events in LMCA FFR-based approach may leave a group of patients at risk of MI, a hypothesis not proven in meta-analysis. FUTURE (FUnctional Testing Underlying Coronary REvascularization) trial ${ }^{16}$ included LMCA disease in $11 \%$ of patients and showed a nonsignificant excess of mortality trend in the FFR group and no clinical benefit of FFR in comparison with angioplasty.

Researchers concluded that in complex, high-risk patients, FFR may not help in treatment decisions and may create a false sense of safety. The European Society guidelines ${ }^{5}$ advocate FFR-guided strategy in absence of ischemia testing (class IA). An important limitation of LMCA FFR is potential overestimation due to presence of downstream lesions. The pressure wire should be advanced distally in both epicardial branches to determine both FFR values by using manual pullback with intravenous (IV) adenosine, and the FFR value in a disease-free daughter vessel should be used. In the presence of downstream lesions, an apparent FFR $>0.85$ in LMCA can be reassuring. ${ }^{17}$ IVUS provides more reliable data through direct lumen visualization, especially in eccentric lesions and in contrast streaming. MLA (minimal luminal area) of $5.9 \mathrm{~mm}^{2}$ and a minimum lumen diameter of $2.8 \mathrm{~mm}$ had the highest sensitivity and specificity correlating with FFR $<0.75 .{ }^{18}$ Park et $\mathrm{al}^{19}$ proposed an MLA cutoff of $4.5 \mathrm{~mm}^{2}$ to predict an FFR $\leq 0.80$ with $77 \%$ sensitivity and $82 \%$ specificity in Asian populations. A deferral strategy may be safe in the short- to medium-term if the IVUS-derived LMCA MLA is $>6 \mathrm{~mm}^{2}$, a cutoff used in the EXCEL trial $(-$ Fig. $\mathbf{1})$.

\section{Clinical Factors}

Presentation with acute coronary syndrome in patients with LMCA disease tips the balance in favor of intervention rather than surgery. In DELTA registry ${ }^{20}$ that included all comers with ULMCA stenosis treated with first-generation DES or CABGs, $13.7 \%$ had acute coronary syndrome. No difference in primary endpoint (death, MI, or stroke) was found between the groups though repeat revascularization was higher in the 

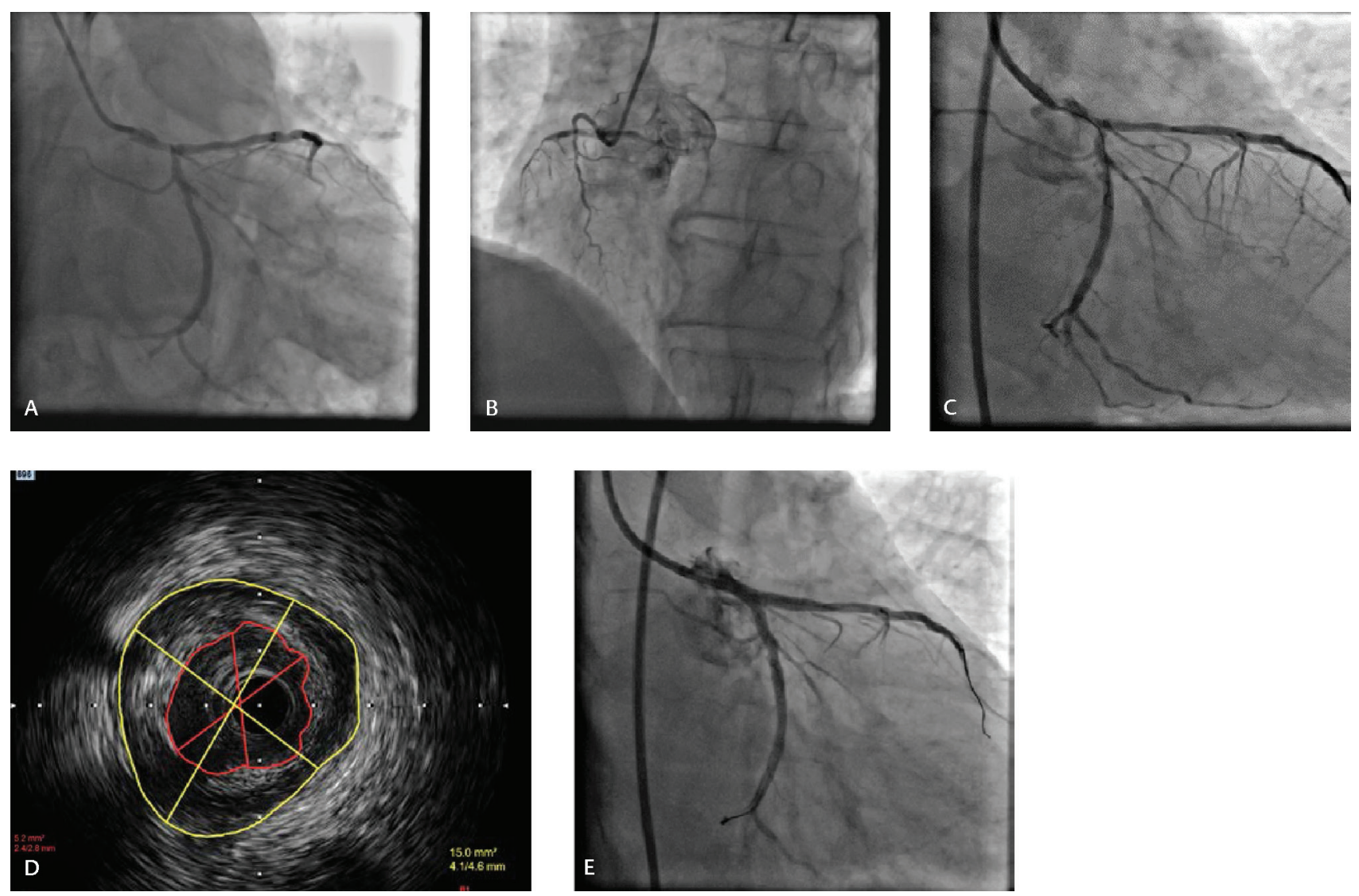

Fig. 1 (A) Baseline CAG revealing significant LM bifurcation complex lesion involving proximal LAD and dominant LCX (Medina 1, 1, 1), (B) Nondominant small RCA. (C) FFR in LAD 0.64, FFR in LCX 0.75 (D) IVUS showing MLA of $5.2 \mathrm{~mm}^{2}$. (E) Final result after two-stent DK crush technique.

PCI group. PCI in patients with ACS and LMCA is proven to be safe despite all comers design and high SYNTAX score (mean SYNTAX $38.0 \pm 18.2$ ).

\section{Anatomic Factors}

The MEDINA classification is an angiographic classification of bifurcation lesion taking into account the plaque distribution into the branches with Medina classes $1,1,1 ; 1,0,1$; and $0,1,1$ denoting true bifurcation lesions. SYNTAX score, SYNTAX II, NERS, and EuroSCORE are widely used to describe anatomic and clinical complexity. The SYNTAX score was incorporated into European guidelines. However, in both EXCEL and NOBLE trials, SYNTAX score was not helpful in predicting outcomes. The DEFINITION (Definitions and impact of complEx bifurcation lesIons on clinical outcomes after percutaNeous coronary IntervenTIOn using drugeluting steNts) ${ }^{21}$ criteria are the only specific risk score for LMCA disease. LMCA lesions are classified as simple if SB diameter stenosis is $<70 \%$ and lesion length $<10 \mathrm{~mm}$. A complex LM lesion has SB diameter stenosis $>70 \%$ and lesion length $>10 \mathrm{~mm}$ or if it satisfies two of the following six minor criteria: (1) moderate to severe calcification; (2) multiple lesions; (3) LAD-LCX bifurcation angle $>70$ degrees; (4) main vessel reference vessel diameter $<2.5 \mathrm{~mm}$; (5) thrombus-containing lesion; and (6) main vessel lesion length $>25 \mathrm{~mm}$.

\section{Technique of Left Main Stenting}

\section{Access and Guiding Catheter}

Radial access with large lumen $6 \mathrm{~F}$ guiding catheters can be used. In case of an aorto-ostial ULMCA lesion, a $6 \mathrm{~F}$ guide catheter is suitable for most of cases, except when debulking is planned and a larger guiding catheter and a stronger support are needed. For very tight aorto-ostial ULMCA lesions or severe damping of the guiding catheter, it is safer to place a guidewire in the guiding catheter, to be able to wire the LMCA LAD or LMCA LCX and promptly disengage the guiding catheter from the LMCA ostium to avoid catheter damping. It is preferable to use a $7 \mathrm{~F}$ guiding catheter in distal LM when elective double stenting is planned or when $>3.5-\mathrm{mm}$ balloons are needed for kissing. The advantages of a $7 \mathrm{~F}$ catheter are better visualization, decreased friction during advancement of the stents, accommodation of all sizes of burr if rotablation is required, and allowing for IVUS to be performed in the presence of multiple guidewires.

\section{Ostial and Shaft Lesions}

The operator must choose the best angiographic view (AP cranial or LAO-cranial) to visualize the ostium of LM and properly position the stent. The implantation of the stent for ostial LM stenosis must be done with a small protrusion into the aorta. After implantation, it is important to post-dilate the stent with properly sized balloons to facilitate good stent apposition at 
the ostium and allow reengagement of the ostium with the catheter (-Fig. 2). The results of LM ostial and shaft lesions are gratifying according to DELTA registry ${ }^{22}$ though firstgeneration DES were used. At 3 years, the rate of major adverse cardiac events (MACE) was $19.1 \%$ versus $28.5 \%$ for distal LM, and the rate of TLR was $4.5 \%$ as against $12.6 \%$ for distal LM.

\section{Distal Lesions}

\section{Wiring}

LAD and LCX should be wired with the most difficult branch wired first. Jailing of the wire in the SB during main vessel stenting is important as it keeps the SB open and is the only marker for rewiring when SB is occluded. The SB wire changes bifurcation angle that facilitates rewiring. Polymer-coated wires were more resistant to retrieval damage and were more efficient in crossing the SB ostium than nonpolymer-coated wires, ${ }^{23}$ but another study reported that jailed polymerjacketed guidewires were associated with procedural MI. ${ }^{24}$

\section{Lesion Preparation}

Lesion is predilated with noncompliant and scoring balloons. Rotational atherectomy is needed for severely calcified LM lesions.

\section{Stenting Strategy-One versus Two Stents}

Several studies have reported that the provisional one-stent approach for distal LM bifurcation was associated with more favorable outcomes compared with two-stent techniques due to lower risk of death, MI, and target vessel revascularization, ${ }^{25-27}$ including a lower risk of stent thrombosis. ${ }^{27}$ A meta-analysis of seven observational studies involving 2,438 patients with mean follow-up of 32 months showed decreased risk of MACE with provisional versus two-stent strategy (20.4 vs. $32.8 \%$; OR: 0.51; 95\% CI: 0.35-0.73), and also decreased target vessel/target lesion revascularization (10.1 vs. 24.3\%; OR: 0.35; 95\% CI: 0.25-0.49) favoring provisional stenting as against two-stent strategy. ${ }^{28}$ In a nonrandomized, retrospective study including all consecutive patients with $50 \%$ stenosis of the LM treated with DES in nine European centers between 2002 and 2004, 62.5\% underwent provisional stenting whereas $37.5 \%$ were in the two-stent group. After 10 years, no differences in TLR or MACE were detected. ${ }^{29}$

\section{Single-Stent Strategy}

European Bifurcation Club (EBC) 12th consensus ${ }^{30}$ recommends a provisional SB stenting approach for LM treatment in most cases (-Fig. 3). It was the strategy used in EXCEL and NOBLE trials with $35 \%$ patients requiring a two-stent strategy in NOBLE trial. A simple lesion defined as SB stenosis $<70 \%$ and lesion length $<10 \mathrm{~mm}$ based on criteria described in the DEFINITION study ${ }^{21}$ favors a provisional approach. Other factors such as a small LCX $<2.5 \mathrm{~mm}$ in diameter or diminutive LCX in a right dominant coronary system and a wide angle between LAD and LCX favor provisional approach.
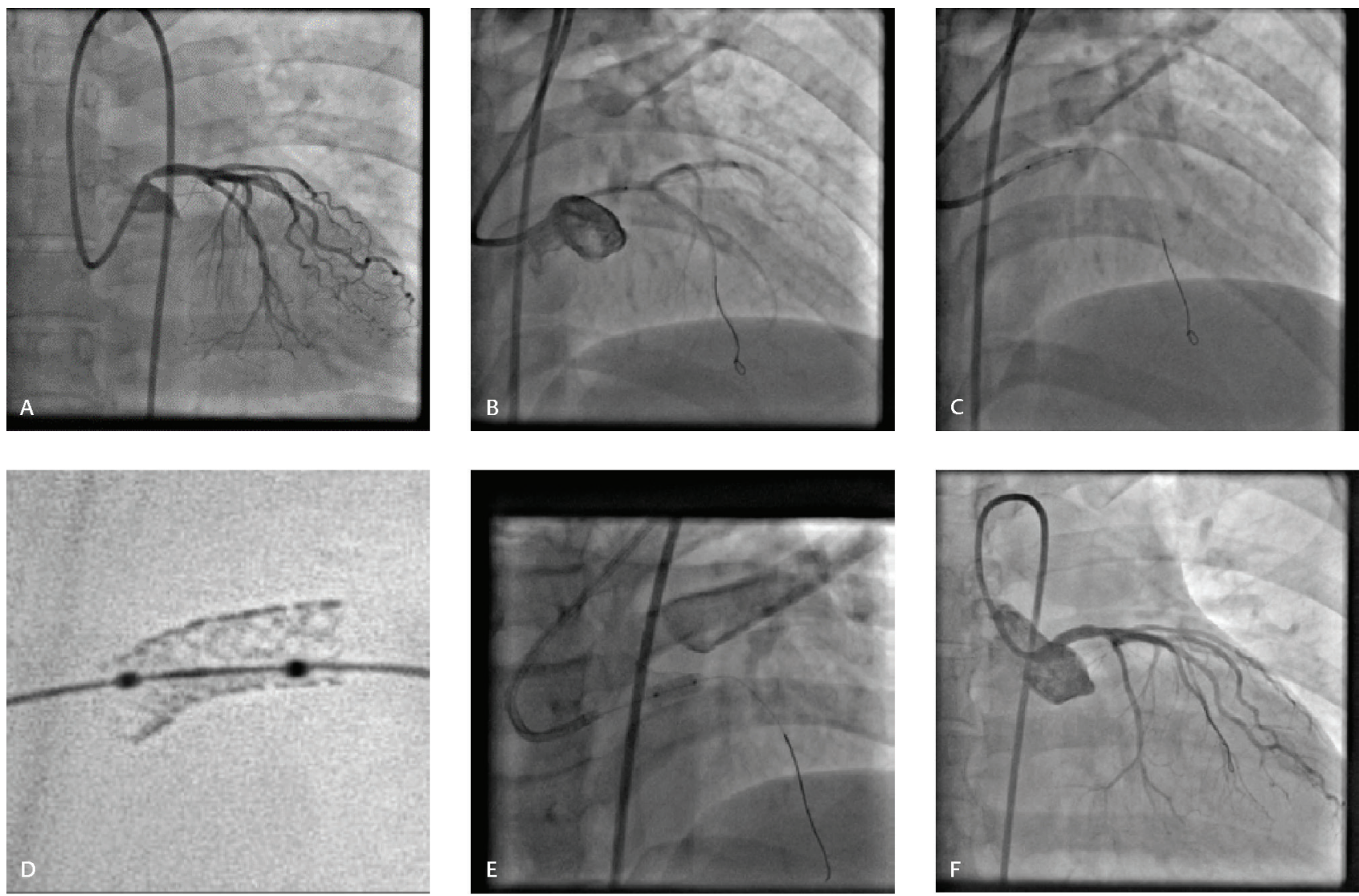

Fig. 2 (A) Left main ostial stenosis in 45-year-old woman with unstable angina. (B) Positioning of stent in left main in AP cranial view. (C) Stent deployment. (D) Stent boost. (E) Post-dilatation of stent (F) Final result. 

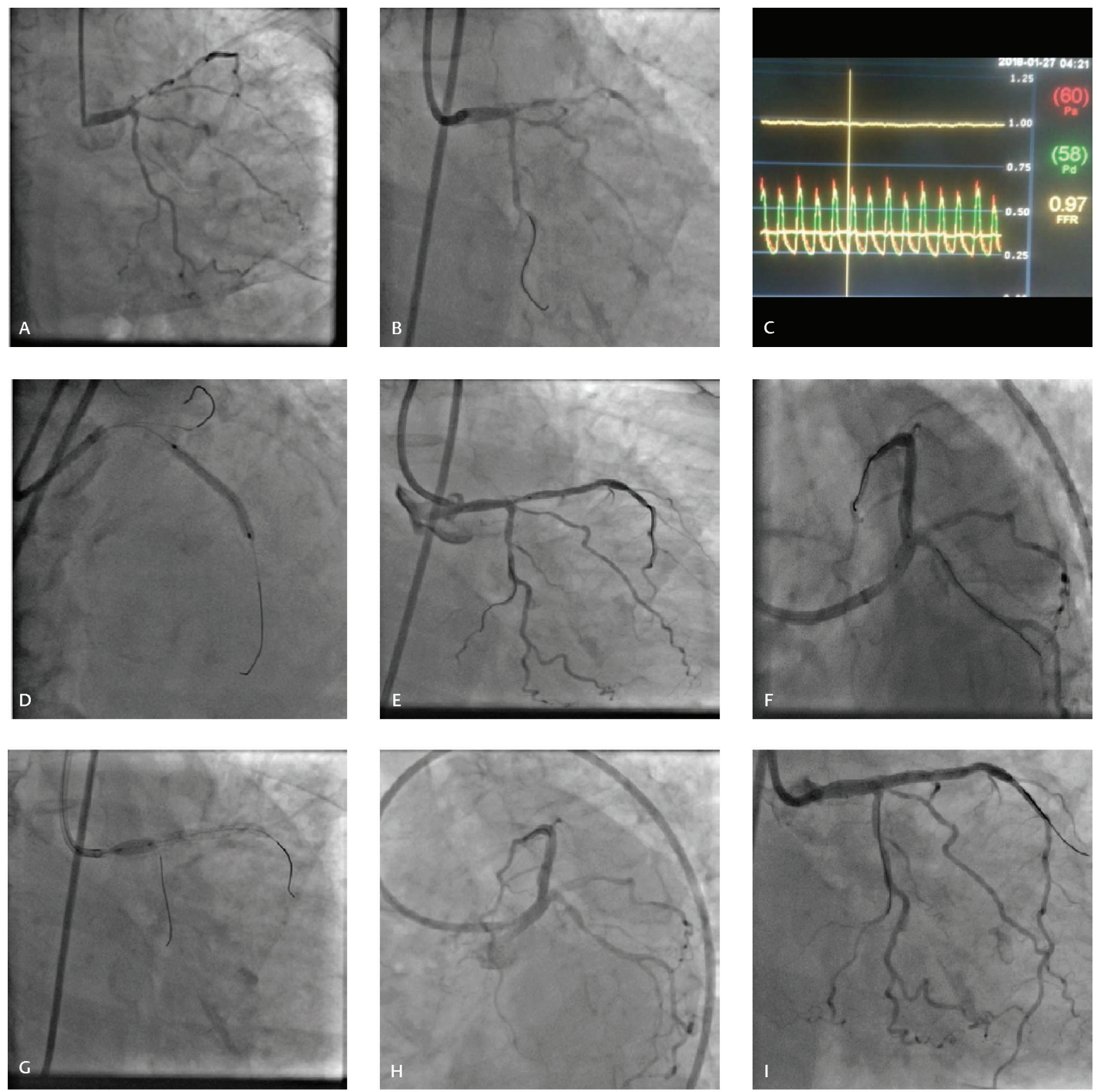

Fig. 3 (A) CAG revealing LM bifurcation lesion involving proximal LAD and LCX beyond ostium (Medina 1, 1, 0). (B) FFR in LCX to decide provisional versus two-stent technique. (C) FFR in LCX 0.97. (D) Mid LAD stenting. (E) Left main to LAD stent. (F) POT balloon marker at carina. (G) Proximal optimization. (H, I) Final result showing good result with no stenosis of ostial LCX.

Second-generation DES should be the first choice. The stent platform should be selected keeping in mind the maximum expansion capability of the stent. The stent size should be selected according to the distal reference diameter to avoid carinal shift. Single-stent crossover from LM into the LAD is the most common approach ( - Fig. 4 ). If the predominant lesion is in the LCX and LAD ostium is spared, provisional stenting from LM toward the LCX can be done (inverted provisional). While planning to stent toward the LAD or LCX, it is important to remember that stenting toward the tightest vessel rather than the largest vessel has better outcomes and reduces the number of stents used. ${ }^{31}$

\section{Proximal Optimization}

Proximal optimization (POT) is performed after stenting by inflating a short balloon just proximal to the carina, to change the tubular stent to a tapered device fitting the LM and distal MB either LAD or LCX, respecting the anatomy of the bifurcation core segment. ${ }^{30}$ Care must be taken so that at least 6 to $10 \mathrm{~mm}$ of stent length is proximal to the carina (the smallest length of commonly available balloons). Careful positioning of the balloon for POT is crucial and may influence the final result: If too distal, it increases the risk of SB occlusion; if too proximal, it has no effect on the stent strut toward the SB. Ideally, the distal shoulder of the balloon should be positioned 
just proximal to the carina while the proximal part is still in the stent to avoid geographical miss. The main problem is that the positioning of the distal marker compared with the distal shoulder varies among the different balloons currently available. Compliant or noncompliant balloons in a 1:1 relation to proximal reference diameter of the LM can be used. POT apposes the stent to LM, reduces ellipticity of stented segment, and prevents accidental abluminal wiring. POT allows strut protrusion into the SB with larger strut opening and minimizes carinal shifting for easier guidewire exchange.

After POT there are three options for the SB ( - Fig. 4):

1. Leaving the $S B$ alone if the result is acceptable.

2. If intervention is required to the $S B, S B$ is rewired through the most distal strut (closest to carina) with either a new wire or by pulling back the MB wire. The jailed wire should be withdrawn carefully to avoid deep intubation of the guiding catheter and consequent damage to the stent. SB is opened with a short noncompliant balloon, and re-POT is done to restore stent distortion opposite the SB. (POT-sidePOT [PSP])

3. After wire exchange, KBI is performed with two short, preferably noncompliant balloons sized according to the distal diameters. Re-POT is performed (POT-KBI-POT).

The clinical outcomes of recent studies comparing KBI versus non-KBI failed to show its advantage over non-KBI treatment in terms of the rate of MACEs, despite lowering SB diameter stenosis at 6 - to 12 -month follow-up. ${ }^{32}$

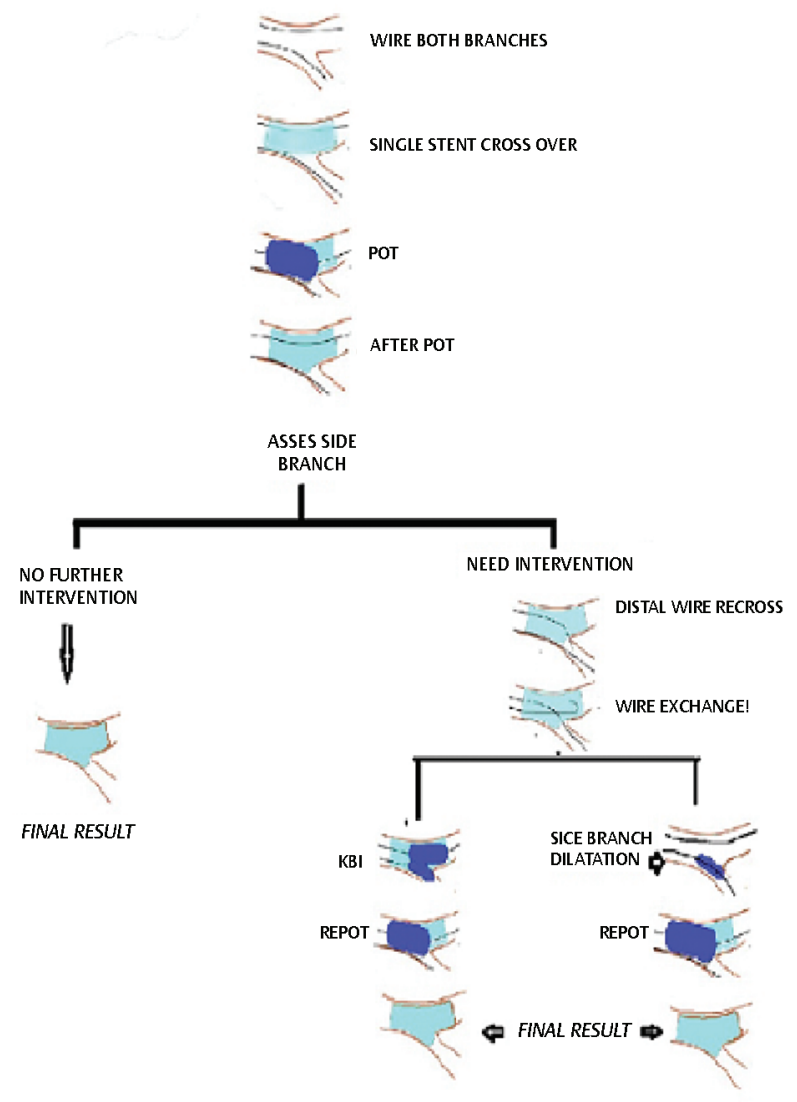

Fig.4 Provisional stenting approach.
According to the Korean Coronary Bifurcation Stenting (COBIS) registry, KBI was associated with a higher MACE rate due to higher TLR rather than death or MI..$^{33}$ The COBIS II registry demonstrated the opposite result with reduced MACE and TLR with KBI. Occlusion of the SB after MV stenting was associated with more frequent cardiac death and MI, which suggested the importance of KBI to secure SB patency ${ }^{34} \mathrm{KBI}$ procedure can be optimized by final POT to correct proximal malapposition, proper guidewire cross in the distal cell to optimize SB strut opening and selecting the appropriately sized balloons for optimizing the geometry. ${ }^{32}$ To reduce proximal deformation, a "modified KBI approach" was recently proposed, using asymmetric inflation pressures: the SB is first inflated to $12 \mathrm{~atm}$, then partly deflated back to 4 atm with simultaneous inflation of the MB balloon at $12 \mathrm{~atm}$. The modified FKBI procedure reduces elliptical stent deformation and optimizes SB access. ${ }^{35}$

\section{Conversion to Two-Stent Strategies}

SB intervention should be performed in patients who develop ECG changes or ischemic symptoms after MV stenting attributable to SB compromise. Treating asymptomatic angiographic stenosis, though done frequently to prevent future deterioration, is controversial. Pilot studies reported a considerable discrepancy between angiographic stenosis (50\%) and FFR values. ${ }^{36,37}$ Incorporating the FFR-guided PCI strategy to treat the LCX reduces the incidence of unnecessary SB intervention and its attendant complications. However, long-term clinical trials are needed to establish FFR-guided SB approach in LM provisional stenting approach. SB stenting can be performed (T, T, and protrusion [TAP] or culotte), with a systematic final KBI and a final POT. If wire recrosses through distal strut, T-stenting is preferred, and if wire crosses through proximal strut, TAP or culotte stenting is preferred ( - Fig. $\mathbf{5}$ ).

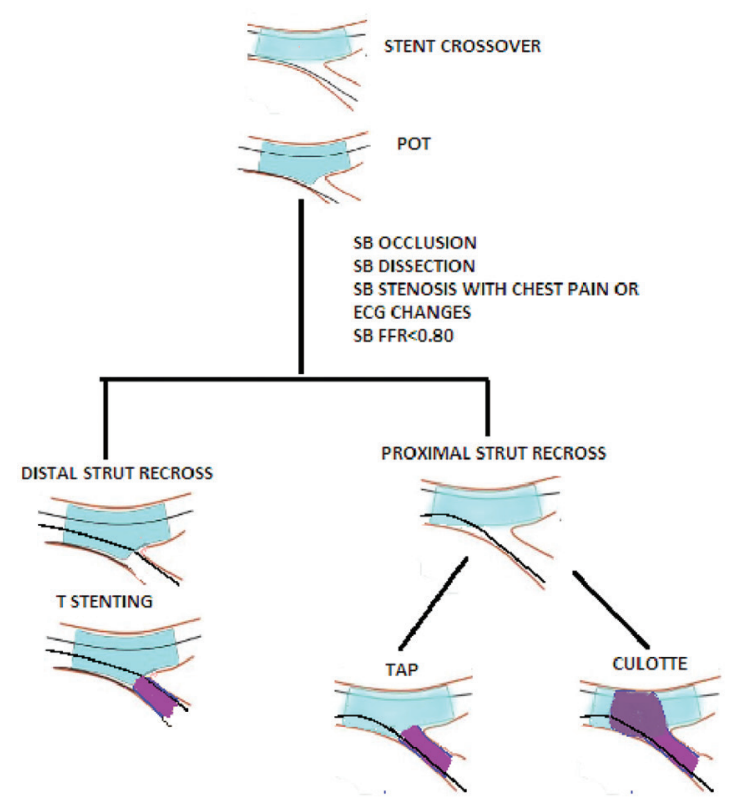

Fig. 5 Provisional stenting conversion to two-stent strategy. ECG, electrocardiogram; POT, proximal optimization treatment; SB, side branch; TAP, T-and-protrusion. 


\section{Two-Stent Techniques}

A complex LMB lesion according to DEFINITION criteria ${ }^{21}$ has SB diameter stenosis $>70 \%$, and lesion length $>10 \mathrm{~mm}$ may necessitate a two-stent technique. This corresponds to Medina classification 1, 1, 1 or 1, 0,1 or 0,1, 1. Large LCX $\geq 2.5$ $\mathrm{mm}$ in diameter or diseased left dominant coronary system also may necessitate two-stent techniques.

T-stenting offers good SB scaffolding with angles $>70$ degrees but could leave a potential gap at SB ostium or protrusion of SB stent into the MB (in the case of TAP). Variation of the T-stenting, the mini-crush technique was developed involving minimal (usually 1-2 $\mathrm{mm}$ ) retraction of the SB stent into the MB before crushing with a stent or with a balloon followed by stent. It is relatively simple, leaves the SB open, and provides good coverage of the SB though it leaves multiple layers of strut. However, due to the low success rate of final KBI and suboptimal long-term outcome in the absence of KBI, this procedure has been modified by Chen et al as the double kissing (DK)-crush technique. ${ }^{38}$

\section{Double Kissing Crush Technique}

This technique consists of stenting the SB, completely crushing the $\mathrm{SB}$ stent with main vessel balloon sized 1:1 to the proximal vessel diameter, proximal SB recross, first KBI, MV stenting, second $\mathrm{SB}$ recross and second $\mathrm{KBI}$, followed by final POT (-Figs. 6, 7). Another key step in the procedure is the alternative inflation with a noncompliant balloon at high pressure $(\geq 16 \mathrm{~atm}$ ) for the SB before each kissing. First kissing can optimize the distorted SB stent, enlarge the cell of the SB stent, and leave only one layer of struts at the ostial SB, which probably facilitates the second kissing after stenting the MV. FKBI was successfully performed in $100 \%$ of cases by DK crush.

\section{Culotte Technique}

There are two distinct culotte techniques. The first technique is part of the provisional strategy. When the result is unacceptable in the SB following provisional stenting, when wire recrosses through proximal strut, or when the angle between LAD and LCX is $<70$, the SB stent is deployed with the proximal part in the LM followed by POT and KBI. ${ }^{30}$ The second strategy ${ }^{39}$ begins with wiring of MV and SB. The SB is then ideally stented first, from before the bifurcation in the MV, to beyond the diseased segment of the SB, with a wire jailed in the MV. After POT, the MV is then rewired through a distal stent strut and the jailed wire is removed. Stent struts are opened with a balloon, and SB wire is removed to prevent metal-to-metal jail and the MV is stented from before the bifurcation to beyond the diseased segment in the MV, according to the diameter of the distal vessel. After a further POT, the SB is rewired, and high-pressure (20 atm) individual noncompliant balloon inflations are made in each vessel at the bifurcation point according to the diameter of the branch vessel to ensure good stent strut separation.

Finally, a lower-pressure KBI is made at the bifurcation. A final POT in stented segment proximal to the bifurcation is optional. The proximal MV is covered by two overlapping stents. It may cause intraprocedural acute closure of the $\mathrm{MB}$ after SB stenting, which can be catastrophic during interventions for distal LM disease. The proximal double stent layers can lead to delayed endothelialization and subsequent stent thrombosis. Finally, the distal MB stent at the ostial LAD artery can be underexpanded because of the positioning through the SB stent strut.

EBC TWO trial ${ }^{39}$ included patients with true bifurcation lesions in which both the main vessel and SB reference diameters were $>2.5 \mathrm{~mm}$ and $\mathrm{SB}$ ostial disease was $\geq 5 \mathrm{~mm}$ in length. There was no difference in the composite endpoint of death, MI, or target vessel revascularization at 12 months between a provisional approach and a systematic culotte stenting. The inclusion of periprocedural biomarker release in composite primary endpoints of trials on bifurcation lesions might favor provisional stenting, though it does not have an independent prognostic significance. A difference in mortality between the two treatment strategies has never been demonstrated, even for bifurcation lesions with large diameter and extension, as in the EBC TWO trial (2.0 vs. $1.1 \%$; $p=0.59$ ). Similarly, in the Nordic-Baltic Bifurcation Study IV ${ }^{40}$ that randomized 450 patents with true bifurcation lesions involving a large SB $(\geq 2.75 \mathrm{~mm})$ to provisional stenting or a two-stent strategy (using the culotte technique in 65.5\% of cases), the rate of cardiac death and all-cause death at 2 years was similar between the two groups. This lack of mortality difference may be due to lack of impact of bifurcation procedures on prognosis, to the low residual risk of patients enrolled in bifurcation trials, to small sample sizes or to the short duration of follow-up. A recent pooled patient-level data analysis from two large bifurcation trials (Nordic bifurcation study and British bifurcation coronary study) with a 5 -year follow-up suggested that a provisional approach is associated with lower all-cause mortality than a systematic dual-stenting technique (3.8 vs. $7 \% ; p=0.04$ ). ${ }^{41}$

DKCRUSH-III study, ${ }^{42,43}$ a randomized multicenter twostent study that compared DK crush with culotte stenting for patients with distal LM lesions, showed increased rates of MACE in patients randomized to the culotte group as opposed to the DK crush group (23.7 vs. $8.2 \%$, respectively; $p<0.001$ ), mainly due to increased MI and target-vessel revascularization. Definite ST rate was 3.4\% in the culotte group and $0 \%$ in the DK crush group. Complex lesions were associated with a higher rate of MACE at 3 years compared with simple lesions, with an extremely higher rate in the culotte group.

The DKCRUSH-V randomized trial ${ }^{44}$ included 482 patients with true distal LM bifurcation lesions (Medina 1,1,1 or $0,1,1)$ to provisional stenting or DK crush stenting. The primary endpoint was the 1-year composite rate of target lesion failure. A planned DK crush two-stent strategy resulted in a lower rate of TLF at 1 year than a PS strategy; $48.3 \%$ of provisional stenting group were transferred into two-stent strategy. One-year TLF was higher in PS group than in DK crush group in complex LM lesions (18.2 vs. $7 \%$; HR 0.27 ; $95 \% \mathrm{CI}$ : $0.05-0.54$ ), thus favoring a DK crush technique in complex lesions. 

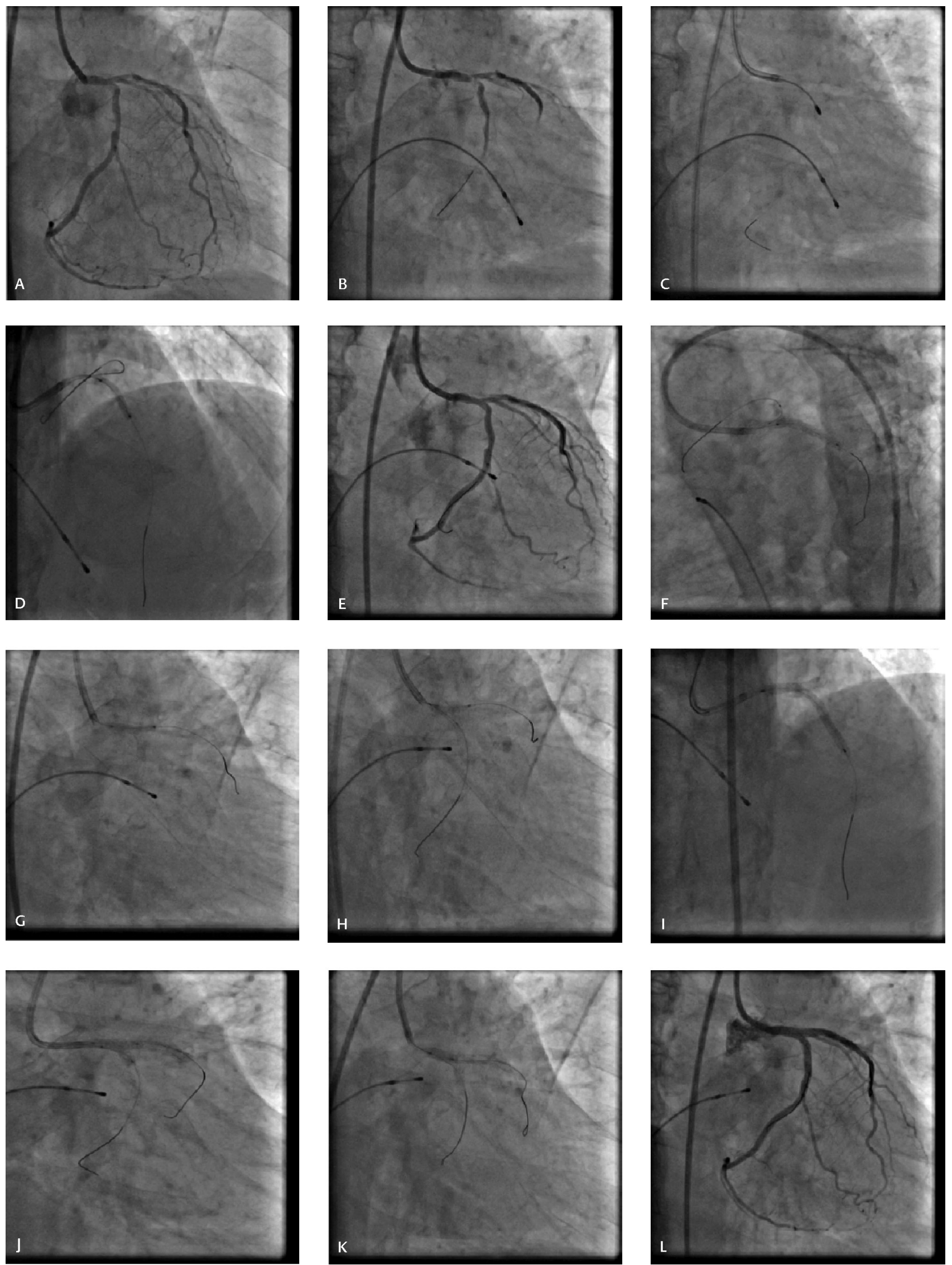

Fig. 6 Double-kiss (DK) crush technique. (A) Baseline CAG revealing significant LM bifurcation lesion involving proximal LAD and LCX (Medina 1, 1, 1) (B) Significant calcification in LM, LAD, and LCX and IVUS catheter did not cross. (C) Rotablation of LCX. (D) Rotablation followed by balloon dilatation of LAD. (E) After adequate lesion preparation. (F) Stenting of LM-LCX. (G) Crushing of LCX stent with noncompliant balloon placed in LM-LAD. (H) First kissing balloon inflation after LCX recross. (I) Deployment of stent in LM-LAD. (J) Second kissing balloon inflation after high-pressure sequential dilatation of LAD and LCX. (K) POT in LM with short noncompliant balloon. (L) Result. 

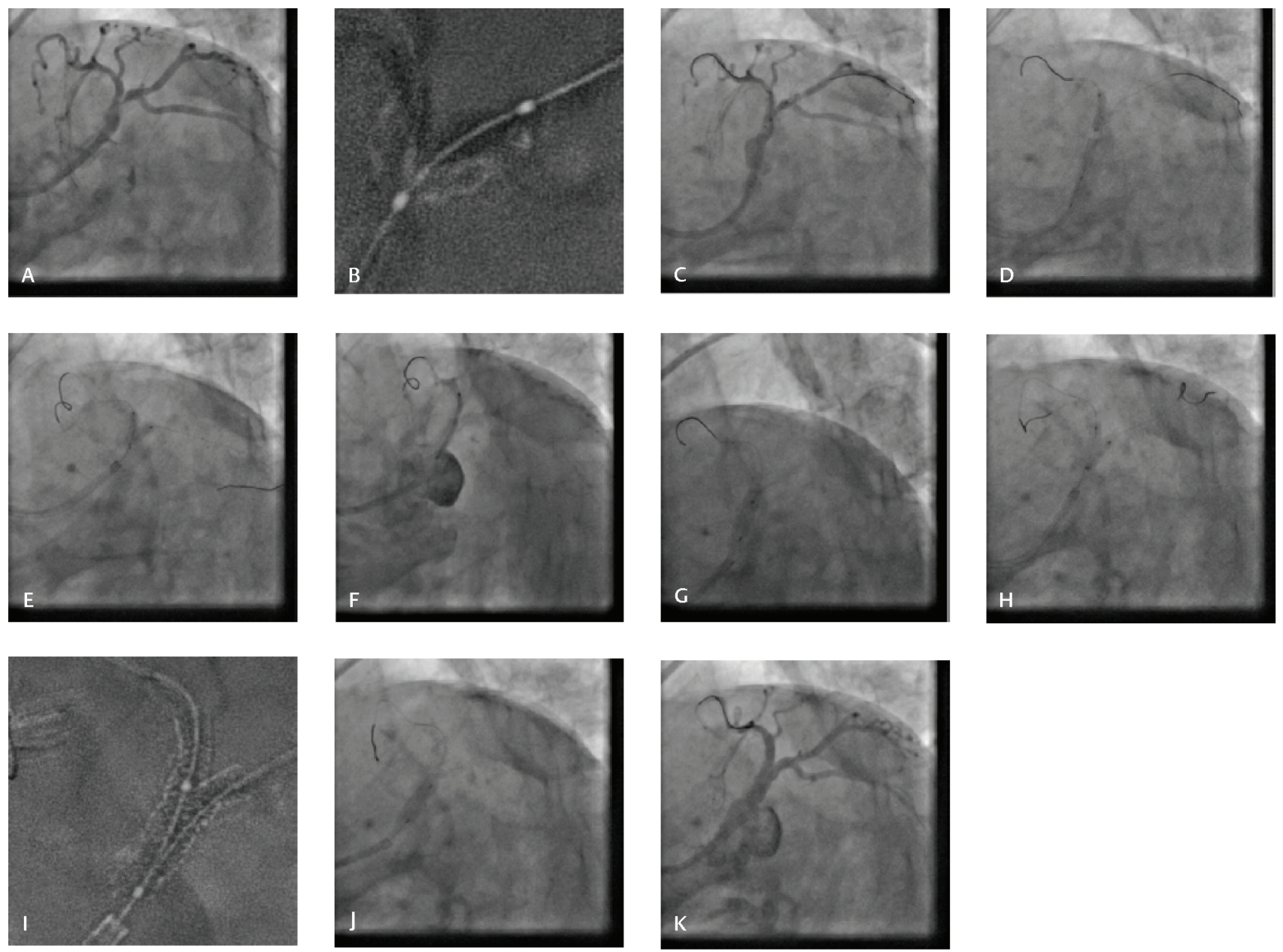

Fig. 7 Double-kiss (DK) crush technique. (A) Baseline CAG revealing significant LM bifurcation lesion involving proximal LAD and LCX (Medina 1, 1, 1), (B) Significant calcification in LM, LAD, and LCX. (C) Stenting of LM-LCX. (D) Crushing of LCX stent with noncompliant balloon placed in LM-LAD. (E) First kissing balloon inflation after LCX recross. (F) Deployment of stent in LM-LAD. (G) POT in LM with short noncompliant balloon. (H) Second kissing balloon inflation after high-pressure sequential dilatation of LAD and LCX. (I) Stent boost. (J) Re-POT. (K) Final result.

\section{Postprocedure Imaging}

FFR post-PCI is used more often to assess the SB to guide intervention in the SB rather than assess the result of stenting LM. Postprocedure imaging with IVUS is strongly advocated. Imaging can identify stent malapposition, dissections, or significant residual disease, which cannot be detected on conventional angiography. Stent underexpansion is the most important cause of DES failure. Kang et al $^{45}$ reported the best IVUS-MSA criteria that predicted angiographic restenosis on a segmental basis included $5.0 \mathrm{~mm}^{2}$ for the LCX ostium, $6.3 \mathrm{~mm}^{2}$ for the LAD ostium, $7.2 \mathrm{~mm}^{2}$ for the polygon of confluence POC, and $8.2 \mathrm{~mm}^{2}$ for the proximal LM above the POC; 33.8\% had under expansion of at least one segment, and angiographic ISR was more frequent in lesions with underexpansion of at least one segment versus lesions with no underexpansion ( 24.1 vs. $5.4 \%, p<0.001$ ).

Two-year major adverse coronary event-free survival rate was significantly lower in patients with underexpansion of at least one segment versus lesions with no underexpansion. A meta-analysis of 10 studies $^{46}$ compared IVUS-guided with angiography-guided PCI to determine the effect of IVUS on the mortality in patients with LMCA disease. IVUS-guided
PCI was associated with significantly lower risks of all-cause death, cardiac death, target lesion revascularization, and in-stent thrombosis. IVUS-guided PCI in LMCA disease significantly reduced the risks of all-cause death by approximately 40\% compared with conventional angiography-guided PCI. Current guidelines recommend IVUS-guided PCI in patients with LMCA disease (class Ila, level of evidence B). ${ }^{5}$ The high spatial resolution of OCT (optical coherence tomography) provides exquisite detail of stent apposition, coverage, and proximal or distal SB guidewire crossing. A shallow depth of penetration, which limits assessment of plaque burden, and the need to flush the lumen to achieve a blood-free field are important limitations in using OCT for optimizing LMCA treatment. Unlike IVUS, there are no comparable data for OCT-guided PCI in the LMCA.

\section{Hemodynamic Support}

Patients with ULM disease with ejection fraction < 35\% constitute a high-risk subset needing hemodynamic support. This was addressed in PROTECT II which compared the Impella 2.5 with an intra-aortic balloon pump (IABP) in patients with an 
average left ventricular ejection fraction of 24\%. The 30-day incidence of MACE was not different for patients with IABP or Impella 2.5 hemodynamic support, though a trend for improved outcome was noted for Impella 2.5 supported patients at 90 days. ${ }^{47}$ The Impella device is recommended for high-risk LM patients with depressed ejection fractions of $<35 \%$.

\section{Conclusion}

PCI for ULM disease using second-generation DES is safe, with clinical outcomes comparable to CABG. The gap between both the techniques has narrowed with the new-generation DES and evolution of techniques of stenting bifurcations. Provisional stenting is the favored approach with two-stent techniques reserved for complex cases. Careful preprocedural planning, systematically performed intervention sticking to the basics of POT, improves outcomes. Imaging further optimizes outcomes.

\section{Conflict of Interest}

None.

\section{References}

1 DeMots H, Rösch J, McAnulty JH, Rahimtoola SH. Left main coronary artery disease. Cardiovasc Clin 1977;8(2):201-211

2 Leaman DM, Brower RW, Meester GT, Serruys P, van den Brand M. Coronary artery atherosclerosis: severity of the disease, severity of angina pectoris and compromised left ventricular function. Circulation 1981;63(2):285-299

3 Taylor HA, Deumite NJ, Chaitman BR, Davis KB, Killip T, Rogers WJ. Asymptomatic left main coronary artery disease in the Coronary Artery Surgery Study (CASS) registry. Circulation 1989;79(6):1171-1179

4 Wykrzykowska JJ, Mintz GS, Garcia-Garcia HM, et al. Longitudinal distribution of plaque burden and necrotic core-rich plaques in nonculprit lesions of patients presenting with acute coronary syndromes. JACC Cardiovasc Imaging 2012;5 (3, Suppl):S10-S18

5 Windecker S, Kolh P, Alfonso F, et al; Authors/Task Force members. 2014 ESC/EACTS Guidelines on myocardial revascularization: the Task Force on Myocardial Revascularization of the European Society of Cardiology (ESC) and the European Association for Cardio-Thoracic Surgery (EACTS) Developed with the special contribution of the European Association of Percutaneous Cardiovascular Interventions (EAPCI) Eur Heart J 2014;35(37):2541-2619

6 Fihn SD, Blankenship JC, Alexander KP, et al. 2014 ACC/AHA/ AATS/PCNA/SCAI/STS focused update of the guideline for the diagnosis and management of patients with stable ischemic heart disease: a report of the American College of Cardiology/ American Heart Association Task Force on Practice Guidelines, and the American Association for Thoracic Surgery, Preventive Cardiovascular Nurses Association, Society for Cardiovascular Angiography and Interventions, and Society of Thoracic Surgeons. J Am Coll Cardiol 2014;64(18):1929-1949

7 Lee PH, Ahn JM, Chang M, et al. Left main coronary artery disease: secular trends in patient characteristics, treatments, and outcomes. J Am Coll Cardiol 2016;68(11):1233-1246

8 Chieffo A, Tanaka A, Giustino G, et al; DELTA 2 Investigators. The DELTA 2 Registry: a multicenter registry evaluating percutaneous coronary intervention with new-generation drug-eluting stents in patients with obstructive left main coronary artery disease. JACC Cardiovasc Interv 2017;10(23):2401-2410

9 Morice MC, Serruys PW, Kappetein AP, et al. Five-year outcomes in patients with left main disease treated with either percutaneous coronary intervention or coronary artery bypass grafting in the Synergy between Percutaneous Coronary Intervention with Taxus and Cardiac Surgery trial. Circulation 2014;129(23):2388-2394

10 Stone GW, Sabik JF, Serruys PW, et al; EXCEL Trial Investigators. Everolimus-eluting stents or bypass surgery for left main coronary artery disease. N Engl J Med 2016;375(23):2223-2235

11 Mäkikallio T, Holm NR, Lindsay M, et al; NOBLE study investigators. Percutaneous coronary angioplasty versus coronary artery bypass grafting in treatment of unprotected left main stenosis (NOBLE): a prospective, randomised, open-label, non-inferiority trial. Lancet 2016;388(10061):2743-2752

12 Nerlekar N, Ha FJ, Verma KP, et al. Percutaneous coronary intervention using drug-eluting stents versus coronary artery bypass grafting for unprotected left main coronary artery stenosis: a meta-analysis of randomized trials. Circ Cardiovasc Interv 2016;9(12):e004729

13 Lefèvre T, Girasis C, Lassen JF. Differences between the left main and other bifurcations. EuroIntervention 2015;11Suppl V):V106-V110

14 Hamilos M, Muller O, Cuisset $\mathrm{T}$, et al. Long-term clinical outcome after fractional flow reserve-guided treatment in patients with angiographically equivocal left main coronary artery stenosis. Circulation 2009;120(15):1505-1512

15 Mallidi J, Atreya AR, Cook J, et al. Long-term outcomes following fractional flow reserve-guided treatment of angiographically ambiguous left main coronary artery disease: a meta-analysis of prospective cohort studies. Catheter Cardiovasc Interv 2015;86(1):12-18

16 Rioufol G, Mewton N, Rabilloud M, et al The FUnctional Testing Underlying Coronary REvascularization (FUTURE) study: a "real-world" comparison of fractional flow reserve-guided management vs conventional management in multivessel coronary artery disease patients. New Orleans, LA: American Heart Association; 2016

17 Fearon WF, Yong AS, Lenders G, et al. The impact of downstream coronary stenosis on fractional flow reserve assessment of intermediate left main coronary artery disease: human validation. JACC Cardiovasc Interv 2015;8(3):398-403

18 Jasti V, Ivan E, Yalamanchili V, Wongpraparut N, Leesar MA. Correlations between fractional flow reserve and intravascular ultrasound in patients with an ambiguous left main coronary artery stenosis. Circulation 2004;110(18):2831-2836

19 Park SJ, Ahn JM, Kang SJ, et al. Intravascular ultrasound-derived minimal lumen area criteria for functionally significant left main coronary artery stenosis. JACC Cardiovasc Interv 2014;7(8):868-874

20 Pyxaras SA, Hunziker L, Chieffo A, et al. Long-term clinical outcomes after percutaneous coronary intervention versus coronary artery bypass grafting for acute coronary syndrome from the DELTA registry: a multicentre registry evaluating percutaneous coronary intervention versus coronary artery bypass grafting for left main treatment. EuroIntervention 2016;12(5):e623-e631

21 Chen SL, Sheiban I, Xu B, et al. Impact of the complexity of bifurcation lesions treated with drug-eluting stents: the DEFINITION study (Definitions and impact of complEx biFurcation lesIons on clinical outcomes after percutaNeous coronary IntervenTIOn using drug-eluting steNts)JACC Cardiovasc Interv 2014;7(11):1266-1276

22 Naganuma T, Chieffo A, Meliga E, et al. Long-term clinical outcomes after percutaneous coronary intervention for ostial/mid-shaft lesions versus distal bifurcation lesions in unprotected left main coronary artery: the DELTA Registry 
(drug-eluting stent for left main coronary artery disease): a multicenter registry evaluating percutaneous coronary intervention versus coronary artery bypass grafting for left main treatment. JACC Cardiovasc Interv 2013;6(12):1242-1249

23 Pan M, Ojeda S, Villanueva E, et al. Structural damage of jailed guidewire during the treatment of coronary bifurcation lesions: a microscopic randomized trial. JACC Cardiovasc Interv 2016;9(18):1917-1924

24 Chatterjee A, White JS, Hashim T, Leesar MA. Jailing polymer jacketed guide-wires during bifurcation coronary interventions is associated with procedural myocardial infarction. World J Cardiol 2017;9(5):442-447

25 Palmerini T, Marzocchi A, Tamburino C, et al. Impact of bifurcation technique on 2-year clinical outcomes in 773 patients with distal unprotected left main coronary artery stenosis treated with drug-eluting stents. Circ Cardiovasc Interv 2008;1(3):185-192

26 Kim WJ, Kim YH, Park DW, et al. Comparison of single- versus two-stent techniques in treatment of unprotected left main coronary bifurcation disease. Catheter Cardiovasc Interv 2011;77(6):775-782

27 Song YB, Hahn JY, Yang JH, et al. Differential prognostic impact of treatment strategy among patients with left main versus non-left main bifurcation lesions undergoing percutaneous coronary intervention: results from the COBIS (Coronary Bifurcation Stenting) Registry II. JACC Cardiovasc Interv 2014;7(3):255-263

28 Karrowni W, Makki N, Dhaliwal AS, et al. Single versus double stenting for unprotected left main coronary artery bifurcation lesions: a systematic review and meta-analysis. J Invasive Cardiol 2014;26(6):229-233

29 D’Ascenzo F, Iannaccone M, Giordana F, et al. Provisional vs. two-stent technique for unprotected left main coronary artery disease after ten years follow up: a propensity matched analysis. Int J Cardiol 2016;211:37-42

30 Lassen JF, Burzotta F, Banning AP, et al. Percutaneous coronary intervention for the left main stem and other bifurcation lesions: 12th consensus document from the European Bifurcation Club. EuroIntervention 2018;13(13):1540-1553

31 Park SJ, Ahn JM, Foin N, Louvard Y. When and how to perform the provisional approach for distal LM stenting. EuroIntervention 2015;11Suppl V):V120-V124

32 Murasato Y, Finet G, Foin N. Final kissing balloon inflation: the whole story. EuroIntervention 2015;11Suppl V):V81-V85

33 Gwon HC, Hahn JY, Koo BK, et al. Final kissing ballooning and long-term clinical outcomes in coronary bifurcation lesions treated with 1-stent technique: results from the COBIS registry. Heart 2012;98(3):225-231

34 Hahn JY, Chun WJ, Kim JH, et al. Predictors and outcomes of side branch occlusion after main vessel stenting in coronary bifurcation lesions: results from the COBIS II Registry (COronary BIfurcation Stenting)J Am Coll Cardiol 2013;62(18):1654-1659

35 Mortier P, Hikichi Y, Foin N, et al. Provisional stenting of coronary bifurcations: insights into final kissing balloon post- dilation and stent design by computational modeling. JACC Cardiovasc Interv 2014;7(3):325-333

36 Kang SJ, Ahn JM, Kim WJ, et al. Functional and morphological assessment of side branch after left main coronary artery bifurcation stenting with cross-over technique. Catheter Cardiovasc Interv 2014;83(4):545-552

37 Nam CW, Hur SH, Koo BK, et al. Fractional flow reserve versus angiography in left circumflex ostial intervention after left main crossover stenting. Korean Circ J 2011;41(6):304-307

38 Chen SL, Zhang JJ, Ye F, et al. Study comparing the double kissing (DK) crush with classical crush for the treatment of coronary bifurcation lesions: the DKCRUSH-1 bifurcation study with drug-eluting stents. Eur J Clin Invest 2008;38(6):361-371

39 Hildick-Smith D, Behan MW, Lassen JF, et al. The EBC TWO study (European Bifurcation Coronary TWO): a randomized comparison of provisional T stenting versus a systematic two stent culotte strategy in large caliber true bifurcations. Circ Cardiovasc Interv 2016;9(9):e003643

40 Kervinen K, Niemelä M, Romppanen H, et al; Nordic PCI Study Group. Clinical outcome after crush versus culotte stenting of coronary artery bifurcation lesions: the Nordic Stent Technique Study 36-month follow-up results. JACC Cardiovasc Interv 2013;6(11):1160-1165

41 Behan MW, Holm NR, de Belder AJ, et al. Coronary bifurcation lesions treated with simple or complex stenting: 5-year survival from patient-level pooled analysis of the Nordic Bifurcation Study and the British Bifurcation Coronary Study. Eur Heart J 2016;37(24):1923-1928

42 Chen SL, Xu B, Han YL, et al. Comparison of double kissing crush versus culotte stenting for unprotected distal left main bifurcation lesions: results from a multicenter, randomized, prospective DKCRUSH-III study. J Am Coll Cardiol 2013;61(14):1482-1488

43 Chen SL, Xu B, Han YL, et al. Clinical outcome after DK crush versus culotte stenting of distal left main bifurcation lesions: the 3-year follow-up results of the DKCRUSH-III study. JACC Cardiovasc Interv 2015;8(10):1335-1342

44 Chen SL, Zhang JJ, Han Y, et al. Double Kissing Crush Versus Provisional Stenting for Left Main Distal Bifurcation Lesions: DKCRUSH-V randomized trial. J Am Coll Cardiol 2017;70(21):2605-2617

45 Kang SJ, Ahn JM, Song H, et al. Comprehensive intravascular ultrasound assessment of stent area and its impact on restenosis and adverse cardiac events in 403 patients with unprotected left main disease. Circ Cardiovasc Interv 2011;4(6):562-569

46 Ye Y, Yang M, Zhang S, Zeng Y. Percutaneous coronary intervention in left main coronary artery disease with or without intravascular ultrasound: a meta-analysis. PLoS One 2017;12(6):e0179756

47 O'Neill WW, Kleiman NS, Moses J, et al. A prospective, randomized clinical trial of hemodynamic support with Impella 2.5 versus intra-aortic balloon pump in patients undergoing high-risk percutaneous coronary intervention: the PROTECT II study. Circulation 2012;126(14):1717-1727 